\title{
Bacterial pathogens causing urinary tract infections in children and their antimicrobial susceptibility patterns in a tertiary care hospital in Sri Lanka
}

\author{
KLW Hathagoda ${ }^{1}$, SS Gunathilaka ${ }^{2}$, B Dissanayake ${ }^{2}$, \\ I Tharaka $^{2}$, K Gunathilaka ${ }^{2}$, R Jayawardane ${ }^{2}$, R Gunapala $^{1}$ \\ Sri Lankan Journal of Infectious Diseases 2018 Vol.8 (2):93-99 \\ DOI: : http://dx.doi.org/10.4038/sljid.v8i2.8222
}

\begin{abstract}
Introduction and Objectives: Urinary tract infection (UTI) is one of the most common infections in childhood. The objective of our study was to ascertain the commonest organisms causing UTI in children and their ABST patterns. The study was carried out in a Teaching Hospital in Sri Lanka.
\end{abstract}

Methods: Data was collected from children who were confirmed to have UTI by positive urine culture ( $>10^{5}$ bacteria/ml urine) between July-December 2015 . The clinical presentation, presence of predisposing factors and reports of urine culture and the antibiotic susceptibility patterns were analysed.

Results: Coliforms were found to be the commonest organism followed by Enterococcus spp. Nitrofurantoin showed the highest sensitivity of the tested antibiotics against both coliforms and the enterococcus groups. However the sensitivity to most of the antibiotics showed a reduction from previously recorded values in studies done in Sri Lanka.

Conclusions: The authors emphasize the need for regular revision of the list of organisms causing UTI and the antibiotic sensitivity to improve the treatment of childhood UTI with the ideal antibiotic.

Keywords: Urinary tract infection, UTI, Children, Pathogens

\section{Introduction}

UTI is one of the most common infections in the paediatric population. ${ }^{1}$ It is a heterogeneous disease which can be categorized into lower and upper UTI, or complicated and uncomplicated UTI, depending on the clinical features and laboratory findings. ${ }^{2}$ It causes significant morbidity and mortality, especially if complicated with structural or congenital defects of the urinary

${ }^{1}$ Lady Ridgeway Hospital for Children, Colombo 8, Sri Lanka

${ }^{2}$ Faculty of Medicine and Allied Sciences, Rajarata University of Sri Lanka, Sri Lanka

Address for correspondence: Dr Shobha Sanjeewani Gunathilaka, Faculty of Medicine and Allied Sciences Rajarata University of Sri Lanka, Sri Lanka Telephone: +94773153361/94711997323

Email: mgrssgunathilaka@gmail.com (D) https://orcid.org/0000-0002-03

Received 4 July 2018 and revised version accepted 29 September 2018

This an open-access article distributed under the terms of the Creative Commons Attribution License, which permits unrestricted use, distribution, and reproduction in any medium, provided the original author and source are credited. 
tract. In children presenting with UTI, 30-40\% have underlying vesico-ureteric reflux (VUR), while other congenital anomalies like posterior urethral valves, pelvi-ureteric junction obstruction, ureteroceles and duplex systems are encountered less frequently. ${ }^{3}$

Fever, increased frequency, straining on micturition, diarrhoea and offensive urine are the common presentations of childhood UTI. ${ }^{3,4}$ Features are often non-specific and it is therefore recommended to perform a semiquantitative urine culture to confirm the diagnosis in children with suspected UTI. ${ }^{5}$ Management of UTI is with either oral or parenteral antibiotics depending on the clinical need. ${ }^{6}$ The initial choice of antibiotics is decided on available clinical guidelines, with availability, cost and personal preferences playing a secondary role. ${ }^{7}$ Clinical guidelines are available in both Sri Lankan and international contexts and provide guidance for initial antibiotic choice. ${ }^{8,9,10}$ As the treatment of the acute episode is of utmost importance, the correct choice of initial antibiotic should be based on local and timely information of the responsible organisms and their ABST pattern. Renal scarring may result from improper treatment, especially in infants and very young children. ${ }^{11}$ Non-response to initial treatment requires a change of antibiotic guided by the ABS results and/or further investigation.

The ABS pattern of a particular organism is known to change with time and vary from place to place, resulting in very specific ABS patterns for causative bacteria in different locations. ${ }^{3,11}$ Probable reasons for varying antibiotic susceptibility include continued exposure of causative bacteria to antibiotics in both clinical and non-clinical settings, including inappropriate and improper use (wrong dose, wrong duration). A positive correlation between antibiotic usage and development of resistance has been observed in many studies. ${ }^{12}$

Information on local ABS pattern is therefore important to treat UTI effectively, while avoiding unnecessary use of a wide range of antibiotics which can contribute to antibiotic resistance. In many countries, updated local information is made available to clinicians for this purpose. However, although the availability of such data in Sri Lanka is increasing, information on causative bacteria and their ABS patterns in children is still limited. ${ }^{13,14}$

Island wide availability of systematic updated information on causative agents/ABS of UTI in the paediatric population is a national need. This study aims to contribute to this need by studying the causative bacteria and their ABS in a paediatric population with confirmed UTI in the North Colombo Teaching Hospital, Sri Lanka.

\section{Methods}

A prospective study was done in the paediatric wards of North Colombo Teaching Hospital during a five-month period from $1^{\text {st }}$ July to $1^{\text {st }}$ December 2015.

Collection of urine for culture was done by mid-stream clean catch sampling. The correct procedure was routinely taught to mothers by one of the medical officers in each ward before collection of urine. Positive urine culture is defined as the presence of significant bacteriuria, (> $10^{5} \mathrm{CFU}$ of a single organism/ml of urine). All the children who were confirmed to have UTI by means of positive urine culture and whose parents consented were included in the study.

An interviewer administered questionnaire was used to obtain the clinical history from the patient's mother or guardian. Thorough clinical examination was performed on the patient by 
the investigators to elicit the signs. Relevant data on the organism and the ABS was extracted from the urine culture reports. The data were entered to a beta version of SPSS statistical software. The frequencies and the cross relationships were analysed where relevant.

\section{Results}

A total of 97 subjects were recruited, 47 (48.5\%) males and 50 (51.5\%) females. The ages varied from two months to twelve years. The mean age was 3.69 years (SD 3.3) (Table 1).

Table 1

Demographic data of the children with culture positive UTI

\begin{tabular}{llrr}
\hline & category & Frequency & Percentage\% \\
\hline Age & $<1$ year & 25 & 25.8 \\
& 1 - 5 years & 44 & 45.4 \\
& $5-10$ years & 18 & 18.6 \\
\multirow{3}{*}{ Gender } & $>10$ years & 10 & 10.3 \\
& Male & 47 & 48.5 \\
& Female & 50 & 51.5 \\
\hline
\end{tabular}

Fever is the commonest presenting feature of UTI in children of all age groups. In $81.4 \%$ of the children with UTI, fever was the main complaint at presentation. Straining, increased frequency of micturition, diarrhoea and offensive urine were the other common complaints (Table 2)

Table 2

Clinical presentations of children with culture positive UTI according to age in the study population

\begin{tabular}{lccccc}
\hline & $<\mathbf{1 ~ y r}$ & $\mathbf{1 ~ - 5} \mathbf{~ y r s}$ & $\mathbf{5 - 1 0}$ yrs & $>\mathbf{1 0}$ yrs & Significance \\
\hline Fever & $84 \%$ & $88.6 \%$ & $77 \%$ & $50 \%$ & 0.04 \\
Straining & $56 \%$ & $45.4 \%$ & $11 \%$ & $10 \%$ & 0.004 \\
Frequency & $28 \%$ & $61.3 \%$ & $55 \%$ & $60 \%$ & 0.054 \\
Dysuria & $44 \%$ & $59.0 \%$ & $88 \%$ & $100 \%$ & 0.001 \\
Offensive urine & $25 \%$ & $15.9 \%$ & $5.5 \%$ & $20 \%$ & 0.308 \\
Diarrhoea & $20 \%$ & $9.1 \%$ & $5.5 \%$ & $10 \%$ & 0.44 \\
Irritability & $32 \%$ & $13.6 \%$ & $0 \%$ & $0 \%$ & 0.12 \\
Abdominal pain & $4 \%$ & $11.4 \%$ & $66 \%$ & $90 \%$ & 0.000 \\
\hline
\end{tabular}

The known predisposing factors for UTI were present in 15 of $97(15.5 \%)$ patients of which constipation and previously diagnosed abnormality of the urinary tract were the most common.

The commonest organisms isolated from urine cultures in children of all age groups were coliforms, accounting for $60.8 \%$ (59/97) of which Klebsiella spp. was found in $9(9.3 \%)$ patients (Figure 1). In 23 patients (23.7\%), enterococci and Staphylococcus spp in $2(2.1 \%)$ patients respectively. There was no gender based difference in the causative organisms (Table $3)$. 
Table 3: Organisms causing UTI in male and female children of the study population

\begin{tabular}{lcccc}
\hline Coliforms & $\begin{array}{c}\text { Enterococcus } \\
\text { spp. }\end{array}$ & Klebsiella & $\begin{array}{c}\text { Staphylococcus } \\
\text { aureus }\end{array}$ \\
\hline Male & $53.3 \%$ & $25.6 \%$ & $10.7 \%$ & $4.3 \%$ \\
Female & $68 \%$ & $22 \%$ & $8 \%$ & $0 \%$ \\
\hline
\end{tabular}

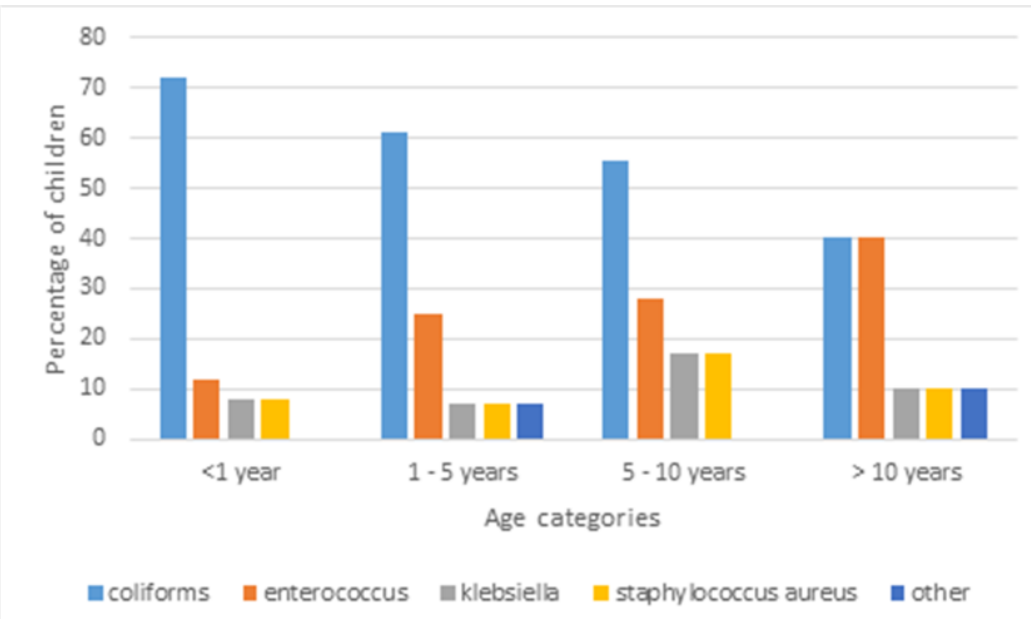

Figure 1: Bacterial pathogens causing UTI in children of different age groups

The organisms causing UTI in different age groups of children is shown in Figure 1. In all age groups coliforms (including Klebsiella spp.) was the commonest isolate. Antibiotics had been started empirically prior to the availability of ABST report in all patients. Co-amoxiclav had been prescribed in 66 of the 97 patients $(68 \%)$ followed by cefotaxime in 15 patients $(15.5 \%)$.

Sensitivity to nitrofurantoin was seen in 40 of the $59(67.8 \%)$ coliforms (excluding Klebsiella spp.) isolated in the study. Sensitivity to nalidixic acid was seen in 34 coliform isolates (57.6\%). Co amoxiclav sensitivity was lower at 52.5\% (31/59 isolates). None of the isolated organisms were found to be ESBL producers. 


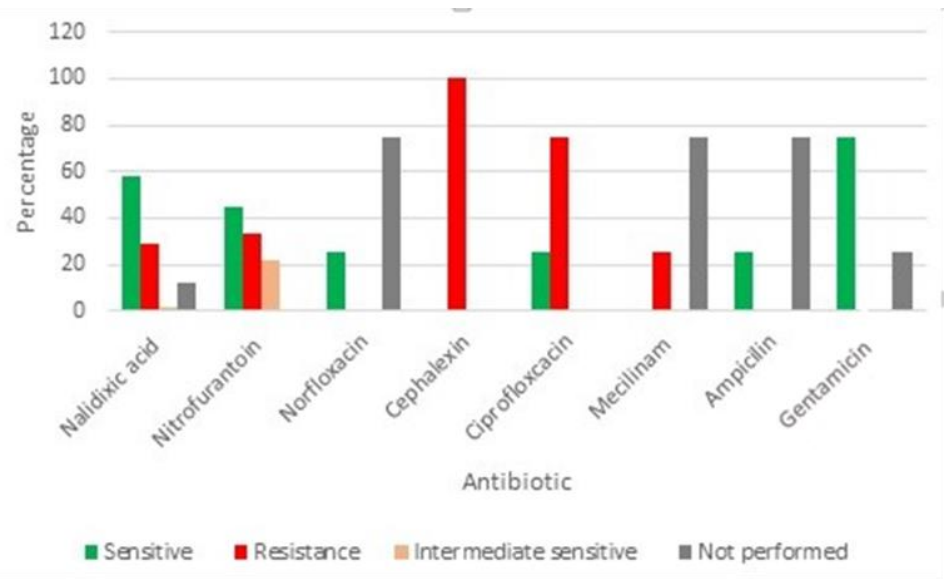

Figure 2 Sensitivity (\%) of coliforms against tested antibiotics

The percentage sensitivity of antibiotics against enterococci were 87\% (20/23), 43.5\% (10/23) and $34.8 \%$ (5/23) for nitrofurantoin, nalidixic acid and co-amoxyclav respectively.

Ampicillin and ciprofloxacin were the least sensitive of tested antibiotics which denotes $14.4 \%$ and $18.6 \%$ respectively for any type of organisms. Figure 2 shows the cumulative percentages of sensitivities of each tested antibiotic against any type of organism.

\section{Discussion}

UTI is a common childhood bacterial infection and accounts for a considerable number of admissions to paediatric wards. The symptoms of UTI vary with age. As previously described by Gunasekara $2010^{7}$, fever was the most commonly encountered symptom and found to be more evident in younger children $(\mathrm{p}=0.04)$ in this study. Dysuria and abdominal pain were seen in older children $(p=0.001)$. These findings could be used in developing clinical criteria for the diagnosis of UTI in children of different age groups. ${ }^{7}$

Coliforms were the most commonly isolated organisms in the current study. However, the isolation rate of $60.8 \%$ in the current study is less than the previously described ${ }^{5}$ Enterococcus spp. was the second most common organism in contrast to previous studies where Klebsiella spp. were identified as the second common infecting organism. ${ }^{5,11}$ It must be noted that although Klebsiella spp. are also 'coliforms', they are often reported independently and are therefore considered in a separate category in the current study.

The enterococci are considered intrinsically resistant to many commonly used antimicrobial agents. ${ }^{16}$ The enterococci have emerged as an important health care associated infectious agent in recent times. Although the children in the study sample did not have a history of prolonged hospital stay or other risk factors for colonization with enterococci, $23.7 \%$ of UTIs in the present study were due to enterococci. The authors believe this may be a negative outcome of the over usage of antibiotics in the community, producing more enterococci with intrinsic and acquired antibiotic resistance. This may need further evaluation in order to prevent more complicated infections with resistant organisms in the future. 
Although co-amoxyclav is the most commonly prescribed antibiotic for empirical treatment of UTI in children, only $52.5 \%$ of coliforms and $34.8 \%$ of enterococci in the study sample were sensitive to this drug. Sensitivity to co-amoxyclav had shown a dramatic reduction from $86.6 \%$ reported by Abeygunawardane e al. ${ }^{12}$ to $52.5 \%$ in the current study. The two studies were done in different locations, Peradeniya and Ragama respectively. No similar studies done in the same study site on a paediatric population was available for comparison. Studies done in adult populations with UTI has detected similar reduction of the susceptibility to antibiotics in Sri Lanka. $^{14}$

A reduction of antibiotic sensitivity is observed all over the world. Overuse, misuse of antibiotics and growing genetic diversity of the organisms are the possible causes for this. ${ }^{17}$ Misuse of antibiotics has been identified as a global crisis since it has given rise to various issues such as increase in antibiotic resistance and spread of Clostridium difficile infections. Discovery of new antibiotics that can combat emerging resistance has been very slow and ineffective. ${ }^{17}$

Collection of the data on antibiotic sensitivity at national and international levels is important to make stakeholders aware of the extent of the problem of antibiotic resistance. Strengthening research on newer drugs is suggested to overcome the crisis of antibiotic resistance ${ }^{18}$ Some countries have reported a reduction in the misuse of antibiotics following interventions including establishment of guidelines for antibiotic use. ${ }^{19}$

\section{Conclusions}

The results of this study emphasize the need for regular surveillance of organisms causing UTI and their antibiotic sensitivity. This information, both local and national, is essential to provide appropriate guidelines for empirical treatment of childhood UTI.

Conflict of interest: The authors declare that there are no competing interests.

Ethics statement: Approval for this study was obtained from the Ethics Review Committee of the College of Paediatricians, Sri Lanka.

\section{References}

1. Donna J Fisher. Pediatric Urinary Tract Infection: Practice Essentials, Background, Pathophysiology. Available at: http://emedicine.medscape.com/article/969643-overview 2016

2. Medical versus surgical treatment of primary vesicoureteral reflux: report of the International Reflux Study Committee. Pediatrics. 1981; 67(3):392-400. PMID: 7017578

3. Ibeneme $\mathrm{C}$, Oguonu $\mathrm{T}$, Okafor $\mathrm{H}$, et al. Urinary tract infection in febrile under five children in Enugu, South Eastern Nigeria. Nig J Clin Pr. 2014;17(5):624-8. doi: 10.4103/1119-3077.141430

4. Schlager T. Urinary tract infections in children younger than 5 years of age: epidemiology, diagnosis, treatment, outcomes and prevention. Paediatr drugs 2001; 3(3):219-27.

doi: $10.2165 / 00128072$

5. Lamabadusuriya SP. A prospective study of urinary tract infections in children. Sri Lanka Journal of Child Health, 2001; 30: 31-6. doi:10.4038/sljch.v30i2.825

6. Gauthier M, Chevalier I, Sterescu A, et al. Treatment of Urinary Tract Infections Among Febrile Young Children With Daily Intravenous Antibiotic Therapy at a Day Treatment Center. Pediatrics 2004;114(4):469-76. doi:10.1542/peds.2004-0421 
7. Gunasekera V. Evidence based management of childhood urinary tract infections. 2010;109:1049. doi:10.4038/sljch.v39i3.2274

8. Urinary tract infection in under 16s: diagnosis and management | Guidance and guidelines |NICE. Available from: https://www.nice.org.uk/guidance/cg54

9. Guidelines n. Management of childhood guidelines on management of. Available from: http://www.slcog.lk/img/guidelines/Other national Gidelines/Paediatricians/Book 01/Management of urinary tract infection in children.pdf

10. Emperical and prophylactic use of antimicrobials- National guidelines 2016:Sri Lanka College of Microbiologists.Available at : http://slmicrobiology.net/download/National-Antibiotic-Guidelines2016-Web.pdf

11. Wani KA, Ashraf M, Bhat JA, et al. Paediatric urinary tract infection: A hospital based experience. J Clin Diagn Res 10(10):SC04-SC07 doi: 10.7860/JCDR/2016/20174.8746

12. Lee YK, Lee H, Kim JM, et al. The antibiotic resistance pattern of Gram-Negative bacteria in children younger than 24 months with a urinary tract infection: A retrospective single-center study over 15 consecutive years. Child Kidney Disease. 2015;19:148-53. DOI: 10.3339

13. Abeyagunawardena AS, Thalgahagoda RS, Pathinayake CA, et al. Antibiotic sensitivity patterns in childhood urinary tract infections. Sri Lanka Journal of Child Health, 2006; 35: 55-60: doi: $10.4038 /$ sljch.v35i2.13

14. Wijekoon C, Dassanayake K, Pathmeswaran . Antimicrobial susceptibility patterns and empirical prescribing practices in adult in patients with urinary tract infection: is there a need for changing clinical practices?. Sri Lankan J Infect Dis 2014;4(1):9-21. doi: 10.4038/sljid.v4i1.6229

15. Howard AJ, Magee JT, Fitzgerald KA, Dunstan FD. Factors associated with antibiotic resistance in coliform organisms from community urinary tract infection in Wales. J Antimicrob Chemother. 2001.47(3):305-13. doi:10.1093/jac/47.3.305

16. Kristich CJ, Rice LB, Arias CA. Enterococcal Infection-Treatment and Antibiotic Resistance. 2014 Feb 6. In: Gilmore MS, Clewell DB, Ike Y, et al., editors. Enterococci: From commensals to leading causes of drug resistant infection. Boston: Massachusetts Eye and Ear Infirmary; 2014. PMID: 24649502

17. Ventola CL. The antibiotic resistance crisis: Part 1: Causes and Threats. Pharmacy and Therapeutics. 2015;40(4):277-283. PMID: 25859123

18. Gould IM, Bal AM. New antibiotic agents in the pipeline and how they can help overcome microbial resistance. Virulence. 2013;4(2):185-191. doi:10.4161/viru.22507.

19. Dan Cui, Xinliang Liu, Peter Hawkey, et al. Use of and microbial resistance to antibiotics in China: a path to reducing antimicrobial resistance. Journal of International Medical Research. 2017; 45(6):1768-1778 doi:10.1177/0300060516686230 